\title{
Dorsal Hand Vein Image Enhancement for Improve Recognition Rate Based on SIFT Keypoint Matching
}

\author{
Sathaporn Chanthamongkol ${ }^{\mathrm{a}}$, Boonchana Purahong ${ }^{\mathrm{b}}$, Attasit Lasakul ${ }^{\mathrm{c}}$ \\ Faculty of Engineering, \\ King Mongkut's Institute of Technology Ladkrabang, Chalongkrung1 Rd., Ladkrabang, 10520, Thailand \\ amr.kolry@gmail.com; byuna001@gmail.com; cklattasi@kmitl.ac.th³
}

\begin{abstract}
The important step in hand vein matching is the quality of image that is significance for hand recognition automatic system. The input image is obvious that bring to high recognize rate important for identifying individual person. in this paper, we will enhance quality of input hand vein image by CLAHE(Contrast limit adaptive histogram equalization) that improve hand vein to clearly and reducing noise by Gaussian and wiener lowpass filter. Keypoint detection, composed by Harris-Laplace is designed to localize keypoint for each image so more discriminative information and then in matching step SIFT keypoint matching. We have investigated the performances using NCUT database. The experimental result shows that the CLAHE is improve keypoint detection, Gaussian and wiener lowpass noise reducing filter is reducing time matching for recognition matching rate base on the SIFT.
\end{abstract}

Keywords-component; regcognition; Keypoint detection; CLAHE; SIFT; Harris detection

\section{INTRODUCTION}

Biometrics are unique physical characteristics, such as fingerprints, iris, face vein that can be used for automated recognition. Biometrics is reliable, convenient and virtually impossible to forge.

Several biometric techniques to identify person and authentication have much variety [1]. In different type of biometric, the vein identification has convenience and is simple in order to use user's hand only. A hand vein can be defined as the subcutaneous vascular pattern on the back of hand [2], and the arrangement of vascular network of human being is stable and unique even between identical twin [3]. It has a high security. It is also extremely difficult to forge. Hand vein exhibits characteristics like uniqueness, stability and has no barriers of culture or gender [4].

It is important for the preprocessing techniques to prepare the data to extract the features of vein patterns. The dorsal hand veins are usually captured by a Near Infrared (NIR) Camera. The vein recognition uses the properties of deoxygenated hemoglobin in venous blood by observing near-infrared righting. The vein images are processed by converted binary images or grayscale images. In binary images, we can use their geometric features (branch points, end points, widths and lengths of vein lines, etc.). In the template matching, we can also use binary images. However, grayscale images are only used in template matching. Thus, we have studied hand vein recognition using binary images.
As a first step, we try the template matching using binary images.

\section{IMAGE ENHANCEMENT}

NCUT is choosing for in this research. The database of hand-dorsal vein images has been built using a vein image acquisition device. It contains 2,040 images of 102 individuals in which 52 are female and 50 are male. The images of each person were captured both left and right hands.

The near-infrared light could easily pass thought the muscle and bone, but absorbed by the hemoglobin in the vein. Some hand vein images acquired by this device are show in Figure1. But size of image for using in processing is too large that unnecessary to processing. On Fig. 1 are sample of images on database, there are low contrast to processing on keypoint matching
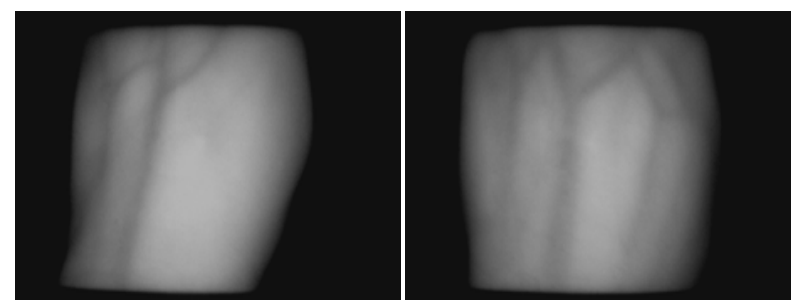

Figure 1. Hand vein image from NCUT Database.
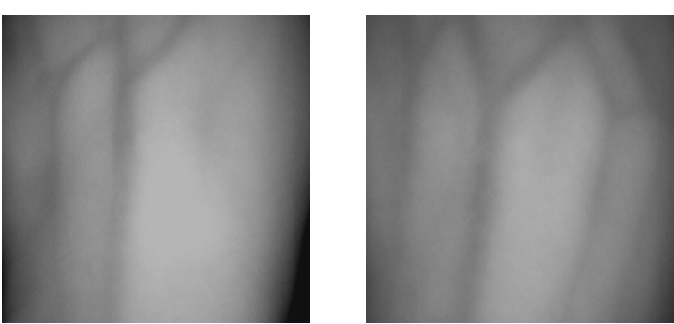

Figure 2. ROI are selected from each images

\section{A. Region of interest(ROI)}

In the vein images, the region that we are interested in is only the region which contains the information of the vein pattern. So we extract the region of interest (ROI) from the 
image. In this work we apply the centroid as the center to the image to extract ROI. The centroid on images $\left(X_{0}, Y_{0}\right)$ of vein images $f(x, y)$ can be calculated as show in (1). And ROI images from equation are result as Fig. 2. The result is selected size $360 \times 360$ px.

$$
x_{0}=\frac{\sum_{i, j} i \times f(i . j)}{\sum_{i, j} f(i . j)} ; y_{0}=\frac{\sum_{i, j} j \times f(i . j)}{\sum_{i, j} f(i . j)}
$$
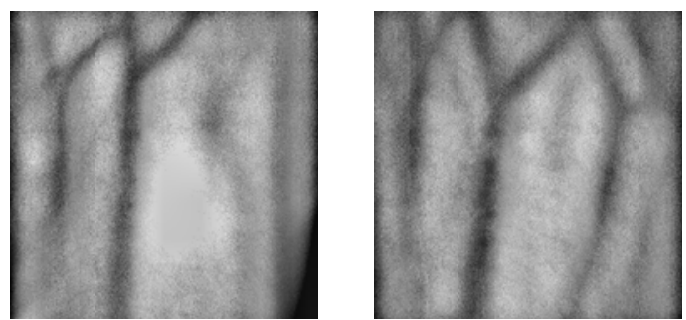

Figure 3. Apply CLAHE to improve contrast of images

\section{B. CLAHE}

Next step of vein image pre processing is to increase the image contrast of ROI to enhance the structure visibility. In this work, A method of contrast of images after ROI selection is low. Therefore, we apply Contrast Limited Adaptive Histogram Equalization (CLAHE) [10] is adopted with the histogram of each block. Some results of this method are shown in Fig. 3.
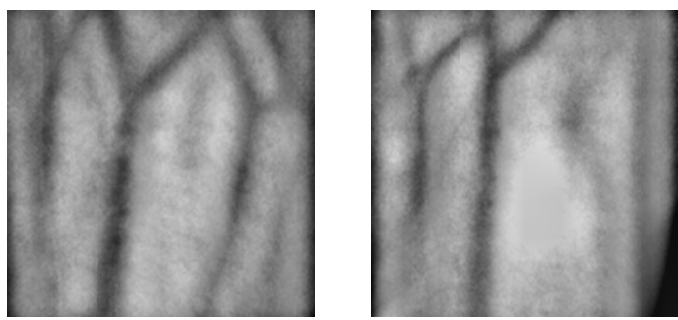

Figure 4. Apply gaussian filter to reducing noise from images

\section{Gaussian filter}

The image after from apply CLAHE to improve contrast of image vein pattern layout on dorsal hand is clearly but there are very high noise on image, therefore we apply Gaussian filter to remove noise on image. Result forms filter show as figure4. From equation two dimensions Gaussians show as (2). The results show in Fig. 4

$$
g(x, y)=\frac{1}{2 \pi \sigma^{2}} \cdot e^{-\frac{x^{2}+y^{2}}{2 \sigma^{2}}}
$$
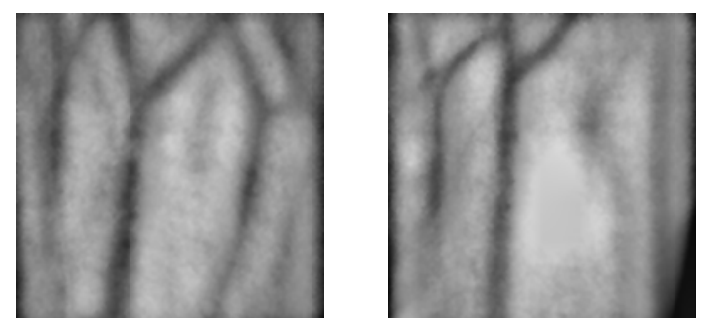

Figure 5. Apply wiener lowpass filter to reducing noise from images

\section{Wiener filter}

Wiener filter is apply to image after Gaussian filter to smoothing image after noise reducing from Gaussian filter result from figure5.

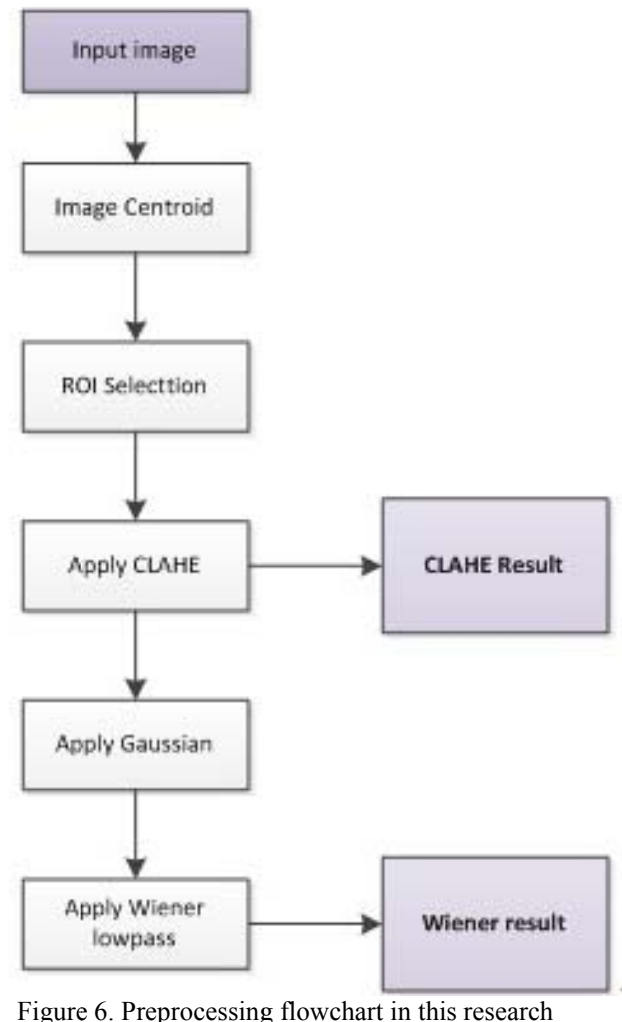

Fig. 6, is a flowchart preprocessing process in this research after result are processed. SIFT keypoint matching are proposed to matching image in this research.

\section{KetPoint Detection \& Sift MAtChing}

Keypoint detection is the primary important step for spare local feature matching, such as SIFT, SURF, A-SIFT etc. firstly of matching ketpoint have to detection on image. In this research keypoint detection is detected by harris detection.

\section{A. Harris keypoint detection}

The harris corner detection algorithm relies on a central principle at corner, the image intensity will change largely 
in multiple directions. This can alternatively be formulated by examining the changes of intensity due to shifts in a local window. Around a corner point, the image intensity will change greatly when the window is shifted in an arbitrary direction. This keypoint detection uses the second moment matrix as the basic of its corner decisions the matrix A, has also been called the autocorrelation matrix and has values closely related to the derivative of image intensity .

$$
A(x)=\sum_{x, y} w(x, y)\left[\begin{array}{cc}
I_{x}^{2}(x) & I_{x} I_{y}(x) \\
I_{x} I_{y}(x) & I_{y}^{2}(x)
\end{array}\right]
$$

Where $I_{x}$ and $I_{y}$ are the respective direction derivatives in $\mathrm{x}$ and $\mathrm{y}$ direction at point $\mathrm{x}$. the corner threshold $\mathrm{A}(\mathrm{x})$ is calculate avoiding the eigenvalue decomposition of the second moment matrix above by equation 4 .

$$
\begin{aligned}
R & =\operatorname{det}(A)-k * \operatorname{tra}^{2}(A) \\
& =\lambda_{1} \lambda_{2}-\alpha\left(\lambda_{1}+\lambda_{2}\right)^{2}
\end{aligned}
$$

Where $\alpha$ is a constant. Corner points have large, positive eigenvalues and would thus have a large Harris measure. Thus, corner points are identified of the Harris measures that are above a specified threshold.
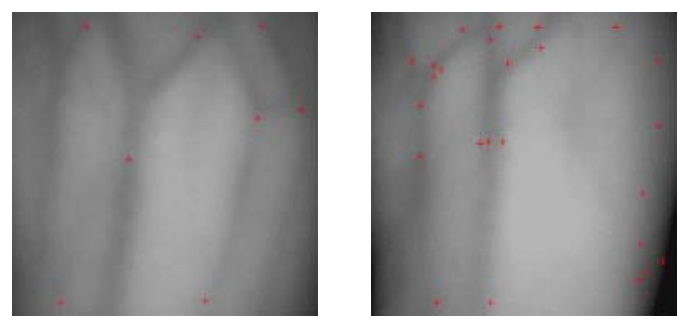

Figure 7. Keypoint detection by harris detection on image before filter
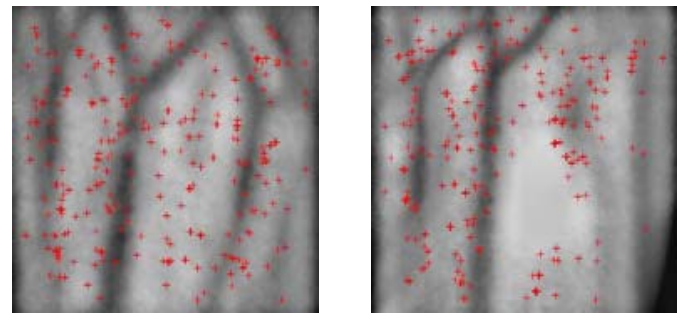

Figure 8. Keypoint detection by harris detection on image after filter

Fig. 7, displays a harris ketpoint detection on ROI image that low contrast and Fig. 8. Show keypoint distribution after enhance image contrast by CLAHE and noise reducing by Gaussian and wiener lowpass filter.

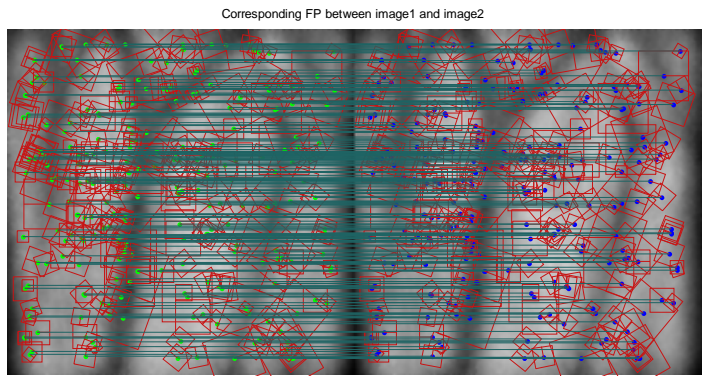

Figure 9. SIFT ketpoint matching on images

\section{B. SIFT matching}

When keypoint are detected SIFT is choose to associate keypoint between differenct dorsal hand vein images due to it's great success in object matching Fig 9.

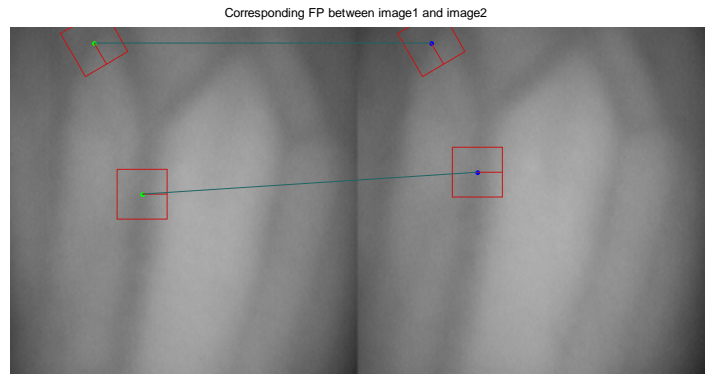

Figure 10. SIFT keypoint matching on same person on ROI images

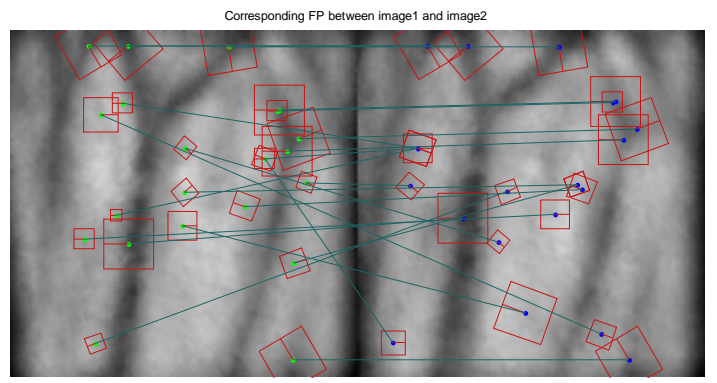

Figure 11. SIFT ketpoint matching on same person after apply filter

SIFT Keypoint matching on Fig. 10 matching is low point due to low keypoint detection by harris. But on Fig. 11 SIFT matching is apply on image after enhance contract by CLAHE and reducing noising by filter that can matching many relative line. 


\section{EXPERIMENT}

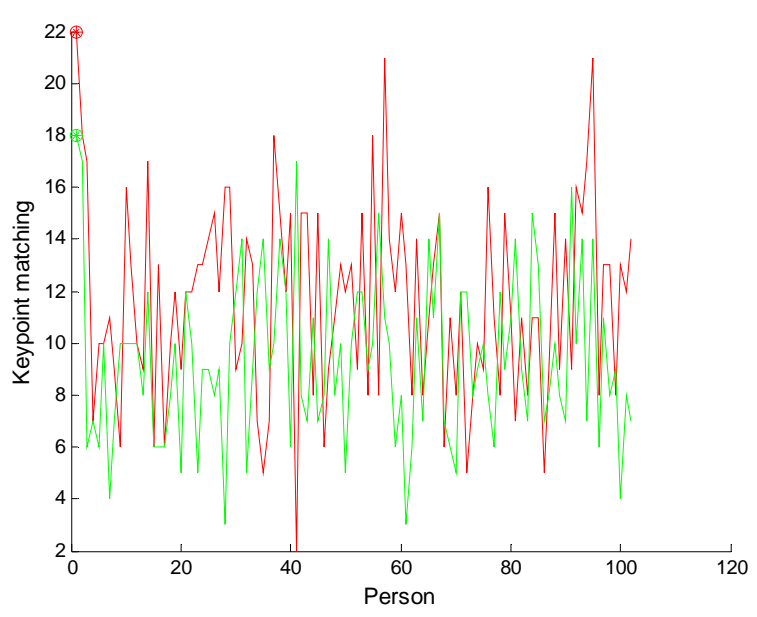

Figure 12. Keypoint matching before and after filter

In this research we choose result image after apply CLAHE (green line) and image after noise reducing by Gaussian filter and wiener lowpass filter (red line). The result as Fig. 12 using SIFT Keypoint matching to maching keypoint between reference image and other image in database, there are 102 person. Figure10 Green line is results from image apply CLAHE and green dot on graph is same person on database, There is highest keypoint more than other on database. And red line is a result after noise reducing and green dot is a number of keypoint matching same person in database.

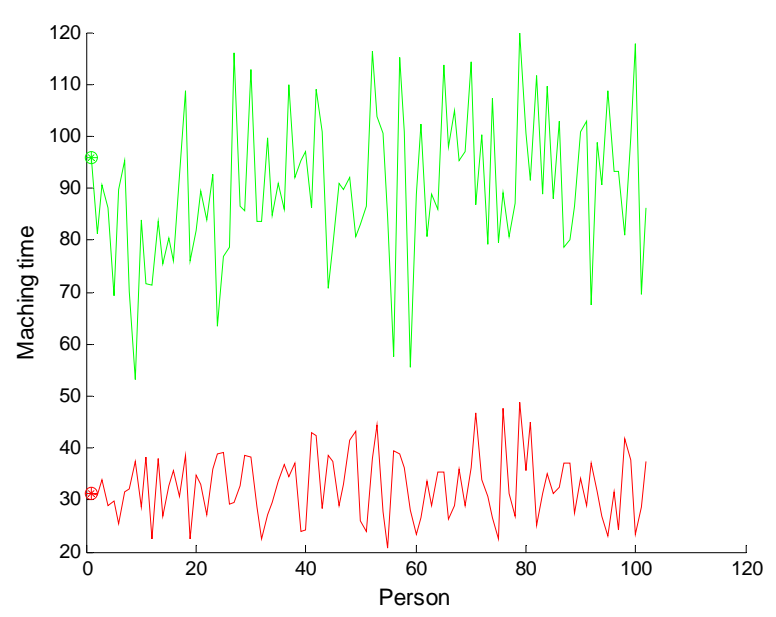

Figure 13. Time of Keypoint matching before and after filter
Not only keypoint matching is improve after reducing noise but maching time is fastest more than before reducing noise after improve contrast by CLAHE in Fig. 13. Show performance of maching time in green line is before noise reducing and red line is time after noise reducing.

\section{CONCLUSION}

In this research CLAHE (Contrast Limit Adaptive Histogram Equalization) is selected to improve contrast of ROI image but result after apply histogram equalization that are many noise in image that there are effect for harris keypoint detection and bring to Keypoint maching to calculate key point that not important. Therefore Gaussian filter, wiener lowpass filter are selected to apply to image after Improve histogram the result after noise reducing is not only higher keypoint maching but processing to calculate keypoint matcing is reducing too.

\section{REFERENCES}

[1] G. Eason, B. Noble, and I. N. Sneddon, "On certain integrals of Lipschitz-Hankel type involving products of Bessel functions," Phil. Trans. Roy. Soc. London, vol. A247, pp. 529-551, April 1955. (references)

[2] J. Clerk Maxwell, A Treatise on Electricity and Magnetism, 3rd ed., vol. 2. Oxford: Clarendon, 1892, pp.68-73.

[3] I. S. Jacobs and C. P. Bean, "Fine particles, thin films and exchange anisotropy," in Magnetism, vol. III, G. T. Rado and H. Suhl, Eds. New York: Academic, 1963, pp. 271-350.

[4] K. Elissa, "Title of paper if known," unpublished.

[5] R. Nicole, "Title of paper with only first word capitalized," J. Name Stand. Abbrev., in press.

[6] Y. Yorozu, M. Hirano, K. Oka, and Y. Tagawa, "Electron spectroscopy studies on magneto-optical media and plastic substrate interface," IEEE Transl. J. Magn. Japan, vol. 2, pp. 740-741, August 1987 [Digests 9th Annual Conf. Magnetics Japan, p. 301, 1982]. 